\title{
Representation of numerical magnitude in math-anxious individuals
}

\author{
Àngels Colomé a,b
}

a Section of Cognitive Processes, Department of Cognition, Development and Educational Psychology, Faculty of Psychology, University of Barcelona, Spain

b Institute of Neurosciences, University of Barcelona, Spain

\section{Correspondence to:}

Àngels Colomé

Section of Cognitive Processes

Department of Cognition, Development and Educational Psychology

Faculty of Psychology

University of Barcelona

Passeig Vall d'Hebron, 171

08035 Barcelona (SPAIN)

Tel. (+34) 933125142

Fax: (+34) 934021363

E-mail: angels.colome@ub.edu 


\begin{abstract}
Larger distance effects in high math-anxious individuals (HMA) performing comparison tasks have previously been interpreted as indicating less precise magnitude representation in this population. A recent study by Dietrich, Huber, Moeller and Klein (2015) limited the effects of math anxiety to symbolic comparison, in which they found larger distance effects for HMA, despite equivalent size effects. However, the question of whether distance effects in symbolic comparison reflect the properties of the magnitude representation or decisional processes is currently under debate. This study was designed to further explore the relation between math anxiety and magnitude representation through three different tasks. HMA and low math-anxious participants (LMA) performed a non-symbolic comparison, in which no group differences were found. Furthermore, we did not replicate previous findings in an Arabic digit comparison, in which HMA individuals showed equivalent distance effects to their LMA peers. Lastly, there were no group differences in a counting Stroop task. Altogether, an explanation of math anxiety differences in terms of less precise magnitude representation is not supported.

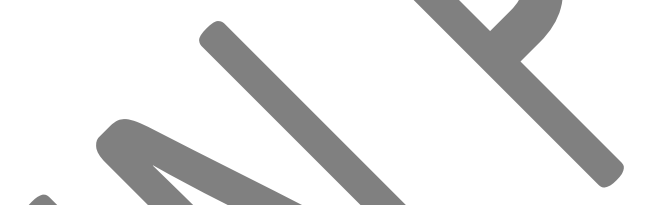

Keywords: math anxiety, distance effect, numerical magnitude, number comparison, counting Stroop 9333 words 


\section{ACKNOWLEDGMENTS}

I would like to thank Julià Agramunt and Maria Roura for their help in preparing the materials and testing participants. I also want to thank M. Isabel Núñez-Peña for her helpful comments. This work was supported by the Spanish Ministry of Economy and Competitiveness and the European Regional Development Fund, FEDER, under grants PSI2012-35703 (MINECO) and PSI2015-69915-R (MINECO/FEDER). Funding was also provided by the Catalan Government (2014SGR-79).

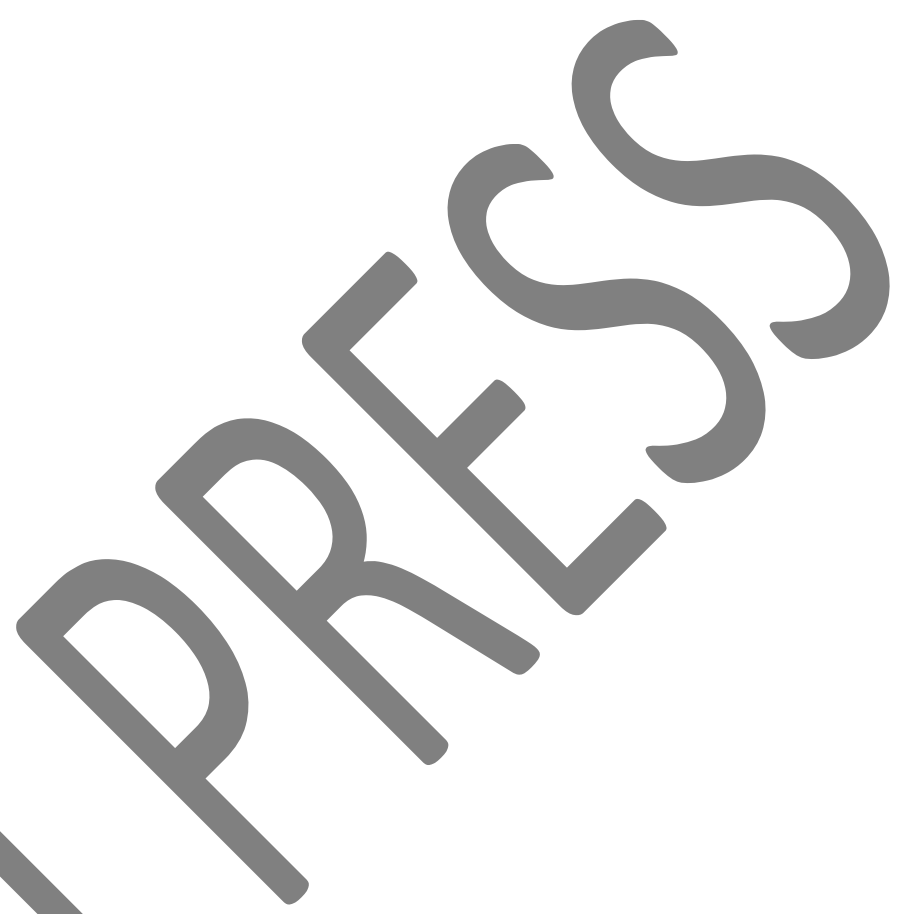


Mathematics anxiety has been defined as a "feeling of tension and apprehension surrounding the manipulation of numbers and the solving of mathematical problems in academic, private and social settings" (Richardson and Suinn, 1972). Both social and cognitive factors seem to play a role in its appearance (for a review, see Chang \& Beilock, 2016; Dowker, Sarkar \& Looi, 2016; Suárez-Pellicioni, Núñez-Peña \& Colomé, 2016). On the cognitive side, three main hypotheses have been proposed. First, math anxiety was initially related to a decrease in working memory capacity caused by intrusive thoughts related to math performance; these thoughts would act as a secondary task taking up resources from the mathematical one (e.g. Ashcraft and Kirk, 2001). Second, it has been proposed (Suárez-Pellicioni, Núñez-Peña, Colomé, 2014) that math-anxious individuals might be more distracted by task-irrelevant stimuli, regardless of whether they are external (task distractors) or internal (e.g. ruminations). Lastly, it has been suggested that mathanxious individuals might have deficient representation of numerical magnitude (e.g. Maloney, Ansari and Fugelsang, 2011). It is generally accepted that numerical magnitudes are represented mentally in the form of an internal mental line ordered by numerical proximity. Close magnitudes are represented by overlapping distributions of activation, which lead to the distance effect: discrimination between two numerosities improves as numerical distance increases. Furthermore, the degree of overlap between the representations of close numbers increases with magnitude, leading to the size effect: for an equal distance, larger pairs of numbers are less discriminable than smaller ones.

Acuity of numerical magnitude improves with age, and it shows individual differences. Maloney, Ansari and Fugelsang (2011) asked participants to conduct a symbolic number comparison task and found an interaction between numerical distance and math anxiety, with high mathematics-anxious individuals (HMA) showing larger distance effects in their latencies than low math-anxious (LMA) peers. They concluded that HMA might represent numerical magnitude less precisely. Several previous studies had reported a link between precision of magnitude representations and current or future high-level mathematical skills (e.g. De Smedt, Verschaffel \& Ghesquière, 2009). Maloney et al. (2011) concluded that people would develop math anxiety because of this difficulty with mathematics. 
Similar results were subsequently obtained by Núñez-Peña and Suárez-Pellicioni (2014)

although in their study the distance x math anxiety interaction was only marginal. However, in both cases, participants had to compare Arabic digits, which might not be the most suitable task for assessing the acuity of internal numerical representation. First, symbolic comparison requires an extra step of processing the symbols and transforming them into an analogous representation. As in another population with low mathematics performance, namely dyscalculia, difficulties might not arise from the approximate magnitude representation itself, but in accessing the numerical magnitude conveyed by the symbols (Rousselle \& Noël, 2007). Second, although it was long considered that magnitudes were represented in an abstract way, more recent evidence indicates that there might be notation-specific representations of numerical quantity in the brain, and symbolic representations might be more precise (Ansari, 2008; Cohen Kadosh, Cohen Kadosh, Kass, Henik \& Goebel, 2007; Piazza, Pinel, Le Bihan \& Dehaene, 2007; Verguts \& Fias, 2004).

All in all, a more direct assessment of magnitude representation in math-anxious individuals was essential. Dietrich, Huber, Moeller \& Klein (2015) tested the acuity of their participants' magnitude representation by asking them to perform a non-symbolic dot comparison task. To compare their results with the previous literature, they also administered an Arabic digits comparison task. HMA and LMA did not differ in their dot comparison performance: there was no significant interaction between the anxiety scores obtained on the Abbreviated Math Anxiety Scale (AMAS) (Hopko, Mahadevan, Bare \& Hunt, 2003) and the latencies, error rates or Weber fraction ${ }^{1}$, which is assumed to be the most direct measure of magnitude representation acuity. In contrast, Dietrich et al. (2015) confirmed the effects of anxiety on the magnitude processing of symbolic information as they observed a larger distance effect in HMA in comparisons of pairs of Arabic digits. However, both HMA and LMA groups had equivalent size effects and performance in terms of latencies or error rates. The combination of larger symbolic distance

\footnotetext{
${ }^{1}$ The Weber fraction is an estimate of noise in the internal magnitude representation. It is considered a reliable index of magnitude representation acuity (Price, Palmer, Battista \& Ansari, 2012), with higher w values implying less precise representations.
} 
effects plus equal performance led the authors to conclude that the distance effect was not due to less precise magnitude representation by HMA individuals, but to impaired comparison processes: since HMA usually tend to avoid working with numbers, their connections between the symbolic representation of numbers and the "which number is larger" response would be weaker than that of LMA. Indeed Van Opstal, Gevers, De Moor and Verguts (2008) had already raised the possibility that distance effects obtained in different tasks could have different loci (see also Van Opstal \& Verguts, 2011, and Verguts, Fias \& Stevens, 2005 for a neural network implementation of this idea). In their study, Van Opstal et al. (2008) used a masked priming paradigm and asked participants to categorize numbers as smaller or larger than a standard, and letters as coming before or after another standard in the alphabet. Their design allowed them to look for distance, congruency priming and priming distance effects. As mentioned before, distance effects refer to longer reaction times for stimuli nearer to the standard, and were found for both numbers and letters. Congruency priming refers to the fact that participants should answer faster in trials in which prime and target elicit the same response (although they only respond to the target). This effect was again found for both letters and numbers. Lastly, priming distance effects show that targets before the standard are answered faster when the prime-target distance is smaller. On this occasion, only number trials showed this effect. Van Opstal et al. (2008) concluded that distance effects could be broken down into a comparison distance effect and a priming distance effect. Comparison distance effects found in letters and numbers would be located at a decisional level. Stimuli connections to the response (e.g. "is larger than") increase monotonically. Close stimuli have similar connection weights to the response nodes and will activate both responses ("smaller than"; "larger than") similarly, causing competition and a delay in the responses. In contrast, priming distance effects would be caused by a representational overlap between the prime and target magnitudes, which occurs for numbers but not for letters. Hence, Van Opstal and Verguts (2011, page 113) concluded that "it is crucial to select a proper task to investigate representational differences or similarities". 
Having observed that distance effects can have two distinct origins, one aim of the current study was to explore the number magnitude representation of HMA and LMA individuals by using a task that could not be solved through a simple comparison network. Specifically, we made use of a counting Stroop task (Pavese \& Umiltà, 1998) in which participants were presented with arrays of identical Arabic digits and had to decide how many digits there were. By manipulating the number of items as well as their identity, we hoped to find distance effects that spoke of the acuity of magnitude representations in both groups.

Moreover, a symbolic and a non-symbolic comparison task were used to compare data with those obtained by the Stroop task in the same sample, and because of some unexpected results that Dietrich et al. (2015) had obtained. Specifically, the symbolic comparison task showed math anxiety differences for the distance effect, but not for the size effect. Both effects were originally considered indicators of access to magnitude representation. However, Verguts and Van Opstal (2005) claimed that they could dissociate (in a same-different task), because the origin of distance effects would be the magnitude representation, and the origin of size effects would be the mapping between these representations and the task-relevant response. As mentioned above, it was subsequently acknowledged that distance effects could also be located at the response level, and this is the alternative that Dietrich et al. (2015) preferred to provide an account of their math anxiety effects. Nevertheless, it seems odd that math anxiety differences at the distance effect that Dietrich and colleagues attributed to response-related factors were not found for the size effect as well. If math anxiety causes individuals to have less experience with numbers and, in consequence, a weaker connection between the symbolic representation and the "which number is larger" response, why does its effect show up when distances are compared but not when sizes are compared? An alternative conclusion might be that only size effects are located at the response level, and the distance effect found in Dietrich et al. (2015) was in the end caused by access to the magnitude representation. Once again, it was necessary to study the locus of the distance effect in which math anxious had differed from their peers by analysing their performance in the counting Stroop. However, it was also important to test the consistency 
of the data gathered by Dietrich et al. in the symbolic comparison task, since they differed from the results of Núñez-Peña and Suárez-Pellicioni (2014) who found that math anxiety affected both the distance and size effects in a symbolic comparison task $(\mathrm{p}=0.056$ and $\mathrm{p}=0.066$ respectively). Notice, though, that the group differences in this last study were only marginal.

To sum up, the aim of this study was twofold. On the one hand, we aimed to replicate Dietrich et al.'s (2015) finding of the absence of math anxiety effects in a dot comparison task: this result would confirm their conclusions rejecting a less precise representation of non-symbolic magnitudes in HMA individuals. We also investigated the representation of symbolic magnitudes using two different tasks. First, we asked our participants to conduct an Arabic digits comparison. Following Dietrich et al.'s (2015) study, we expected to obtain differences caused by math anxiety. We also manipulated both distance and size in an attempt to confirm the pattern they had obtained for the two effects. In order to check that the differences in the distance effect across math anxiety groups were located at the response level, we also conducted a counting Stroop task and studied the effect of the distance between the number of items (response) and the identity of the Arabic digits presented (distractor). This distance effect is considered to be caused by an overlap in the magnitude representation. Hence, if differences between math anxiety groups were also obtained, they should be attributed to worse acuity of numerical representation in HMA individuals. Otherwise, the hypothesis of a task-response level origin of the distance effect in symbolic comparisons would be reinforced. In what follows, we will describe the method used. We will first report the aspects common to the three experiments and then detail the specific features of each one of them.

\section{Method}

\section{Participants}

Fifty-six participants took part in this study, and were divided into a high math-anxiety (HMA) and a low math-anxiety group (LMA). They were selected from a sample of 490 university students from the University of Barcelona's Faculty of Psychology who were assessed for math 
anxiety and trait anxiety at introductory psychology courses and who constitute the database of a larger project.

Participants were divided into two groups according to their scores on the Shortened Mathematics Anxiety Rating Scale. Participants in the two groups were paired according to their scores on the trait subtest of the State-Trait Anxiety inventory so as to ensure that trait anxiety was equivalent across groups. Trait anxiety and math anxiety are correlated (Hembree, 1990). Furthermore, trait anxiety has been related to differences in dynamic adjustment of attentional control (e.g., Eysenck, Derakshan, Santos, \& Calvo, 2007). On both the nonsymbolic and counting Stroop tasks participants had to deal with some level of conflict between task-relevant and task-irrelevant stimuli (perceptual information and identity of the numbers to be counted respectively). Therefore, it was important to ensure that any differences that emerged between groups could not be explained by this yariable.

The LMA group was comprised of 28 participants (26 women and two men, age range $=19-24$; mean $=20.71 ; \mathrm{SD}=1.43$ ) who scored below the first quartile (score range $=35-56$ mean $=47.85$; $\mathrm{SD}=5.54$ ) in the Shortened Mathematics Anxiety Rating Scale (sMARS) (Alexander \& Martray, 1989). The HMA group also comprised 28 participants (27 women and one man, age range $=19-27$, mean $=21.17, \mathrm{SD}=2.29)$ who scored above the third quartile (score range $=78-$ 104; mean=85.53; $\mathrm{SD}=6.88$, on the sMARS.

Groups differed in math anxiety, $t(54)=22.54, p<.001$, but not in trait anxiety (mean=17.92; $\mathrm{SD}=7.19$ and mean $=20.89, \mathrm{SD}=7.81$ for the LMA and HMA respectively), $t(54)=1.47, p=.14$, age, $t(54)<1$, or gender distribution, $\chi^{2}(1)=0.35, p=.55$.

\section{Materials}

Screening phase. Shortened Mathematics Anxiety Rating Scale (sMARS) (Alexander \&Martray, 1989) The sMARS is a 25 -item version of the Math Anxiety Rating Scale (MARS) (Richardson \& Suinn, 1972), which measures math anxiety by presenting 25 situations that may cause it (e.g., being given a homework assignment of many difficult problems, which is due in the next class). A five-point Likert scale ranging from 1 (no 
anxiety) to 5 (high anxiety) can be used by respondents to describe their level of anxiety associated with each item. Thus, total scores for the instrument range from 25 to 125 . The Spanish version of the sMARS was used (Núñez-Peña, Suárez-Pellicioni, Guilera \& Mercadé-Carranza, 2013). This version has strong internal consistency (Cronbach's alpha $=.94)$ and high 7-week test-retest reliability (intra-class correlation coefficient $=$ .72). The internal consistency of the sMARS in the current study was Cronbach's alpha $=.95$.

\footnotetext{
State-Trait Anxiety Inventory (STAI) (Spielberger, Gorsuch, Lushene, Vagg \& Jacobs, 1983) The STAI is a 40-item scale that can be used to measure state (STAI-S) and trait (STAI-T) anxiety, although in this study only trait anxiety was assessed. The STAI-T score reflects a general and relatively stable tendeney to respond with anxiety. The score is obtained by presenting respondents with 20 items describing different emotions and asking them how they feel "in general". Items are answered on a fourpoint Likert scale ranging from 0 (almost never) to 3 (almost always). The Spanish version used in this study shows good to excellent internal consistency (Cronbach's alpha $=.95)$, and adequate 20 -day test-retest reliability with college students $(r=.86)$ (Spielberger, Gorsuch \& Lushene, 2008). The internal consistency of the STAI-T subscale in the current sample was Cronbach's alpha=.82.
}

Experimental session. The materials used during the experimental session will be described in each experiment section.

\section{General Procedure}

All participants undertook three tasks, namely, non-symbolic comparison, Arabic digits comparison and counting Stroop. The order of the tasks was randomised across participants in an equivalent way for the two groups. 
Participants were tested individually. When they entered the experimental room they were asked for some demographic information, informed about the general procedure of the session and asked to sign a consent form. Instructions for each task were provided immediately before they conducted it. All of them were paid for their participation.

\section{Experiment 1A: Non-symbolic Comparison}

\section{Materials and Procedure}

A version of Panamath v1.22 (the Psychophysiological Assessment of Numerical Approximation, www.panamath.org; Halberda, Mazzocco \& Feigenson, 2008) was used. Participants were presented with spatially separated arrays of blue and yellow dots and asked in which colour were there more dots. Each colour always appeared in the same position of the screen and participants had to answer by pressing a keyboard button ( $\mathrm{F}$ or $\mathrm{J})$ corresponding to the side with more dots. In half of the cases there were more blue dots, and in the other half yellow dots were more numerous. Stimuli were displayed for $500 \mathrm{~ms}$. The number of dots in each colour ranged from 5 to 20 . The ratio was manipulated to vary the difficulty of the task. The full set of ratios used was: 1.1, 1.2, 1.3, 1.5, 1.8 and 2.0. Since participants might have based their answers on perceptual variables rather than the number of dots, dot size was manipulated: in half of the trials the total area of each colour was proportional to the number of dots (non-size-controlled), but in the other half, the total area of the dot set with less dots was larger than the area of the dot set with more dots (anti-correlated trials). By mixing ratios and congruency, 12 different combinations were obtained. Eight trials of each combination were presented for a total of 96 trials. Two more trials were used as training and were not considered in the subsequent analyses. This task lasted approximately 7 minutes. Latencies and the percentage of correct responses were recorded for each participant. Furthermore, Panamath software calculated the Weber fraction $w$, which is an estimate of noise in the participants' internal magnitude representation. 


\section{Results and Discussion}

Participants' performance was first analysed in terms of latencies and accuracy (percentage of correct responses). Trials with erroneous responses were removed. Latencies that deviated more than two standard deviations from the participant mean were also discarded. Table 1 shows mean response times (RTs) and the percentage of hits for each ratio, congruency and math anxiety group.

Insert Table 1 about here

Repeated measures ANOVA were conducted with the ratio $(1.1 ; 1.2 ; 1.3 ; 1.5 ; 1.8$ and 2$)$ and congruency of the perceptual and numerical information (non-size-controlled vs. anti-correlated trials) as within-participant variables, and math anxiety as the between-participant variable.

HMA and LMA groups did not differ in their reaction times, $F(1,54)<1$. Latencies showed a main effect of ratio, $F(5,270)=54.63, p<.001, \eta_{\mathrm{p}}^{2}=50$, with reaction times decreasing as the ratio between the two magnitudes increased. Paired comparisons showed significant differences $(p<.05)$ between all ratios, with the exception of the pair 1.1 vs. $1.2, t(54)<1$. Ratio interacted with math anxiety, $\mathrm{F}(5,270)=4.05, p=.007, \mathrm{y}_{\mathrm{p}}^{2}=.07$. Visual inspection of the data showed that latencies were longer for LMA participants than for their HMA peers in the smaller ratios but that this pattern inverted from ratio 1.5 onwards. Although group differences were not significant at any ratio (all $t \mathrm{~s}(54)<1$, except ratio 2 : $t(54)=1.002 ; p=.32$ ) we conducted an additional analysis in which we compared responses to successive ratios for each group This analysis confirmed an interaction between ratio and math anxiety for the comparison of ratios 1.3 vs $1.5, F(1,54)=5.73, p=.020 \mathrm{y}_{\mathrm{p}}^{2}=.09$, as well as for the 1.8 and 2 ratios, $F(1,54)=4.40$, 
$p=.04, \mathrm{y}_{\mathrm{p}}^{2}=.07^{2}$. None of the other comparisons reached significance (all $p \mathrm{~s}$ for ratio $\mathrm{x}$ math anxiety interaction >.20).

There was also a main effect of congruency: as expected, participants answered faster in trials in which perceptual and numerical information were correlated than in those in which they were not, $F(1,54)=14.40, p<.001, \eta_{\mathrm{p}}^{2}=.21$. Moreover, an interaction between ratio and congruency was obtained, $F(5,270)=6.84, p<.001, \eta_{\mathrm{p}}^{2}=.11$ : significant regular congruency effects were only found at ratios 1.3, 1.8, and 2 respectively, $t(55)=2.53, p=.01 ; t(55)=6.43, p<.001$ and $t(55)=2.69, p=.009$, while, unexpectedly, there was an advantage for incongruent trials at ratio $1.1, t(55)=2.31, p=.025^{3}$. Math anxiety did not interact with congruence, $F(154)<1$. The triple interaction ratio x congruency $\mathrm{x}$ math anxiety did not reach significance either, $F(5,270)<1$. Nevertheless, given that our interest focused primarily on the effects of math anxiety, it was essential to verify the strength of the null results obtained. We therefore used the JASP software (JASP Team, 2016; jasp-stats.org) to calculate the Inclusion Bayes Faetor (BF inclusion) for matched models ${ }^{4}$, for each of these interactions as well as for the main effect of anxiety. BF inclusion reflects the change from prior to posterior inclusion odds for a factor of interest by comparing all models that contain the relevant factor to equivalent models without it. We obtained an Inclusion Bayes Factor of .55 for math anxiety, confirming that the current data provide scarce support for the inclusion of this factor. As for the interactions, BF inclusion for

\footnotetext{
${ }^{2}$ This last significant interaction can be attributed to the fact that latencies for LMA participants abruptly descended between ratio 1.8 and ratio 2 in the incongruent trials, $t(27)=4,21, p<.001$. In contrast, no ratio effects were obtained for the HMA group or in the congruent trials of LMA participants (both $p s>.20$ ). ${ }^{3}$ There is no universally accepted best procedure for generating stimuli that control for non-numerical features; different parameters are used across the published studies (De Wind \& Brannon, 2016). When using the Panamath software to generate our stimuli, we opted not to control the convex hull or the total "envelope area" taken up by the dot arrays. According to Clayton, Gilmore and Inglis (2015) experiments in which the convex hull is not controlled have sometimes led to an absence of any congruency effect, or even to more accurate responses for the incongruent trials. Even if we did find a significant congruency effect, we cannot completely rule out the possibility that the observed ratio $\mathrm{x}$ congruency interaction was due to the fact that some of the trials of the ratios where no effect of congruency was found had a larger convex hull in the dot sets with a lower cumulative surface area of the dots.

${ }^{4}$ Until recently BF inclusion was not usually reported; BF10 was mostly used. BF10 reflects how likely the data are under the alternative hypothesis compared to the null hypothesis. However, in cases like ours, the complex design leads to an explosion of models to compare. More importantly, we were mainly interested in interactions, and the interaction models that the BF10 compares with the null hypothesis also comprisethe main effects of each of the variables. Hence, a high BF10 for the interaction model might instead be exclusively due to a relevant effect of one (or both) main factors. To correct for this, and in response to the suggestion of S. Mathôt, the most recent JASP versions have implemented the Inclusion Bayes Factor for matched models described above.
} 
math anxiety $\mathrm{x}$ congruency was .13 and the triple interaction obtained a BF inclusion value of .02. Thus, Bayesian analyses provided new evidence against an interaction of math anxiety with congruency or distance $\mathrm{x}$ congruency.

The accuracy analyses replicated most of the findings obtained for latencies, although math anxiety showed no effect at all. Accuracy increased with ratio, $F(5,270)=98.17, p<.001, \mathrm{\eta}^{2}{ }_{\mathrm{p}}=.64$. All paired comparisons showed significant differences $(p<.04)$. Furthermore, fewer errors were committed when perceptual and numerical information led to the same answer, $F(1,54)=42.61$, $p<.001, \mathrm{y}_{\mathrm{p}}^{2}=.44$. Once again, there was a significant interaction between ratio and congruency, $F(5,270)=5.28, p<.001, \mathrm{y}_{\mathrm{p}}^{2}=.089$. When each ratio was analysed separately, only the ratios of $1.2,1.5$ and 1.8 showed significant differences between the two congruency conditions (respectively, $t(55)=4.16, p<.001 ; t(55)=3.48, p=.001$ and $t(55)=6.97, p<.001)$. Lastly, there were no differences between the two groups, $F(1,54)<1$, and math anxiety did not interact with any variable $(F<1$ for all interactions, including the triple one).

Once again we conducted extra Bayesian analyses to check the solidity of the lack of significance obtained through the frequentist statistics. BF inclusion for math anxiety had a value of .17 , confirming the irrelevance of this predictor. As for the ratio $\mathrm{x}$ group, congruency $\mathrm{x}$ group and ratio x congruency $\mathrm{x}$ group interactions, BF inclusion values were, respectively, .02, .14 and .02. Thus, we found no evidence that math anxiety affected participants' accuracy in this task. To conclude, $w$ fractions were also analysed. As expected, a congruency effect showed more precision in the responses in which perceptual cues helped participants, $F(1,54)=27.18$, $p<.001$,

$\mathrm{y}_{\mathrm{p}}^{2}=.33$. However, there were no differences in the acuity of the magnitude representation of HMA and LMA nor an interaction between anxiety group and congruency (both $F \mathrm{~s}(1,54)<1)$. On this occasion $\mathrm{BF}$ inclusion values were .39 for anxiety and .27 for the interaction between anxiety and congruency, if anything, arguing against the inclusion of anxiety as a relevant factor. 
To sum up, despite finding the expected effects for other variables such as ratio or congruency between perceptual and numerical information, we failed to observe differences caused by math anxiety in a non-symbolic comparison task. Both high and low math-anxious individuals took a similar time to respond and performed the task with equivalent accuracy. When we specifically analysed the acuity of their magnitude representation, no differences were observed either. Moreover, groups behaved equivalently at all the ratios and math anxiety did not interact with congruency. Therefore, there does not seem to be an association between math anxiety and the acuity of the internal representation of quantities. Our results confirm the data obtained by Dietrich et al. (2015) and reject the way that Maloney et al. (2011) interpreted the differences they obtained in their symbolic comparison task: a deficient amodal representation of numerical magnitude does not seem to be the basis of the worse performance of high math-anxious in an Arabic digits comparison.

Experiment 1B: Symbolic Comparison

Materials and Procedure The same material as in Rousselle \& Noel (2007) was used here. Pairs of one digit numbers ranging from 1 to 9 (Courier New, 32) were presented in each trial and participants had to decide which number was the largest. The size and distance between the numbers were manipulated, leading to four different conditions (see Table 2): small numbers (1-5), close distance (1); large numbers (5-9), close distance (1); small numbers (1-5), large distance (3-4) and large numbers (5-9), large distance (3-4). Three different items were used in each condition and they were presented six times: three in ascending order (larger number on the right side) and three in descending order. Thus, a total of 72 items were presented ( 3 digit pairs $\times 2$ sizes $\mathrm{x} 2$ distances $\mathrm{x} 2$ orders $\mathrm{x} 3$ presentations). Items were pseudo-randomised by ensuring that the same item did not appear in two consecutive trials and that there were no more than three trials in a row requiring the same answer. Eight more trials were used as practice before the experiment started. 
Insert Table 2 about here

Each trial had the following structure: first, an asterisk appeared centred on the screen for 500 ms. Subsequently, the asterisk was replaced by the two digits presented simultaneously, one beside the other. The digits remained on the screen until an answer was provided, or for a maximum of $3000 \mathrm{~ms}$. Participants had to press the "D" key if the largest number appeared on the left, and the " $\mathrm{K}$ " key if the right digit was the largest one. Lastly, a blank of $500 \mathrm{~ms}$ was left between trials.

\section{Results and Discussion}

Latencies and accuracy rates were analysed. Before conducting the latency analysis, all trials that had been completed erroneously or with latencies deviating more than two standard deviations from each participant's mean were discarded. Table 3 shows the mean RTs and percentage of correct responses for each math anxiety group and condition.

Insert Table 3 about here

A repeated measures ANOVA was performed with size and distance as withinparticipant variables and math anxiety as a between-participants factor. The reaction times of HMA and LMA groups did not differ, $F(1,54)<1$. There was a main effect of distance, $F(1,54)=201.80, p<.001, \eta^{2}=.78$. However, distance effect did not interact significantly with math anxiety, $F(1,54)=1.44, p=.23, \mathrm{y}^{2}{ }_{\mathrm{p}}=.026$. A main size effect was also observed, $F(1,54)=167.16, p^{<.001,} \mathrm{y}^{2}=.75$, which did not interact with math anxiety, $F(1,54)<1$, either. Lastly, there was a significant distance $\mathrm{x}$ size interaction, $F(1,54)=17.57, p<.001, \mathrm{y}_{\mathrm{p}}^{2}=.24$, which was not affected by math anxiety $(F(1,54)<1$ for the triple interaction)._Given that previous studies had found an interaction between math anxiety and distance (e.g. Dietrich et al, 2015) or size (e.g. Núñez-Peña \& Suárez-Pellicioni, 2014), it was important to assess the strength of our 
results. We again ran Bayesian analyses and obtained $\mathrm{BF}$ inclusion values of 0.48 for the distance $\mathrm{x}$ math anxiety interaction, of 0.19 for the size $\mathrm{x}$ math anxiety model, and of 0.25 for the triple interaction distance $\mathrm{x}$ size $\mathrm{x}$ math anxiety. Hence, since the data reduced the prior probability of these models, Bayesian analyses confirmed the non-significant effects obtained in our previous frequentist results. Subsequently, we conducted similar analyses on accuracy rates. Groups did not differ in their number of hits, $F(1,54)=2.68, p=.10, \mathrm{y}_{\mathrm{p}}^{2}=.04$. A distance effect was found again, with smaller distances causing more errors, $F(1,54)=43.89, p<.001$, $\mathrm{y}_{\mathrm{p}}^{2}=.44$, but this effect did not interact with math anxiety, $F(1,54)<1$. There was also a significant size effect, $F(1,54)=71.65, p<.001, \mathrm{y}^{2}=.57$, which did not interact with math anxiety, $F(1,54)=1.002, p=.32, \mathrm{y}_{\mathrm{p}}^{2}=.018$, either. In contrast, distance and size interacted significantly, $F(1,54)=35.52, p<.001, \mathrm{y}_{\mathrm{p}}^{2}=.39$. Lastly, the triple interaction distance $\mathrm{x}$ size $\mathrm{x}$ math anxiety was not significant, $F(1,54)<1$._Again, Inclusion Bayes Factors were calculated for each relevant interaction, providing the following values: distance $\mathrm{x}$ math anxiety, $\mathrm{BF}$ inclusion $=.23$; size $\mathrm{x}$ math anxiety, $\mathrm{BF}$ inclusion $=.30$ and distance $\mathrm{x}$ size $\mathrm{x}$ math interaction, $\mathrm{BF}$ inclusion $=0.37$. Thus, once more, the interactions did not receive support from our data. To sum up, we failed to replicate previous findings of larger symbolic distance effects on the latencies of high math-anxious participants: Therefore, our results diverge from the findings of Dietrich and collaborators (2015): HMA and LMA participants' performance in a symbolic comparison task did not differ, analysing either size or distance effects. Dietrich et al. (2015) claimed that the differences between math anxiety groups that they found when measuring distance effects were located at the connection between the symbolic representation and the response. Given that our main interest in this study was to explore the acuity of magnitude representation in math anxious participants, it was still possible that other tasks more clearly tapping at this level of processing would show group differences. To test this possibility, we ran a last experiment using a counting Stroop paradigm: participants had to decide how many Arabic digits appeared on the screen, while ignoring their identity. It has repeatedly been found that when there is a discrepancy between the number of items displayed and their identity, 
participants are slower than in congruent or neutral (e.g. naming non-numerical symbols)

conditions. Furthermore, the size of the interference depends on the arithmetic difference

between the symbols and the number of them (Hock \& Petrasek, 1973; Pavese \& Umiltà, 1998).

This effect led Pavese et al. (1998) to conclude that both the vision of a digit and the

enumeration process would autonomously activate the same magnitude representation,

organised as a compressed number line. Hence, we hypothesised that if a distance effect was

replicated, and if group differences were found in it, they would both be located at the level of

magnitude representation. This would go against the conclusion by Dietrich et al. (2015), since

it would show that high and low math-anxious individuals (also) differ in the precision of the

magnitude representation they access from symbols. In contrast, if the group differences in the distance effect they found in the number comparison disappeared here, the explanation by

Dietrich et al., based on comparison processes, would be reinforced.

Materials and Procedure Experiment 1C: Counting Stroop

Participants were presented with numerosities between 1 and $4^{5}$. Three conditions were created.

In the congruent condition, the identity of the Arabic digits to be counted was consistent with the enumeration response (e.g. 22). Each numerosity was presented 9 times. In the incongruent condition (e.g. 11), the result of the enumeration process could diverge from the identity of the elements counted by 1,2 or 3 : therefore, three distances were presented. Again, each numerosity was presented on 9 occasions, 3 with each Arabic digit (e.g. for the answer "four", the elements displayed could be 1111,2222 or 3333).

\footnotetext{
${ }^{5}$ Small numerosities were selected because they were expected to produce faster and more homogenous responses. However, the numerosities used were within the subitising range. Subitising refers to a fast, effortless apprehension of numerosity without the need for counting. It has been widely debated whether subitising reflects the functioning of the object tracking system, a mechanism devoted to tracking small sets through time and space, or the general magnitude representation system. Pavese and Umiltà (1998) used numbers that belonged to the subitising range (1-5) and to the counting range (5-9) in a betweenparticipants design. The effect of symbolic distance was similar in both groups. Pavese and Umiltà (1998) claimed that the distance effect in the subitising range was similar to that obtained in priming or comparison tasks, and concluded that it was associated with activation of the same magnitude representation.
} 
Lastly, in the neutral condition, stimuli were randomly selected as uppercase letters F, R and J. Each letter appeared on three occasions for each numerosity. In total, 108 trials were presented in a pseudo-randomised order with the following restrictions: no more than two consecutive trials were carried out requiring the same response, and no more than two trials of the same condition were undertaken in a row. A block of twelve neutral trials was used to familiarize participants with the task. All of them contained letters different from the ones used in the experiment, and were not further analysed. Each trial followed this structure: first, an asterisk was displayed for $500 \mathrm{~ms}$. Then, the stimuli to enumerate were presented for $4000 \mathrm{~ms}$ or until a response was provided. Lastly, a blank of $1000 \mathrm{~ms}$ was left between trials.

Participants were asked to answer the number of symbols, ignoring their meaning. They had to provide their answer via a computer keyboard: answers 1, 2, 3, and 4 corresponded respectively to the $\mathrm{C}, \mathrm{V}, \mathrm{N}$ and $\mathrm{M}$ keys. Participants were instructed to place their fingers over the keys throughout the experiment.

\section{Results and Discussion}

One participant in the LMA group was not included in these analyses because she was given a previous version of the experiment by mistake.

The incongruent condition required participants to enumerate digits that differed from the quantity to name by 1 (18 trials), 2 (12 trials) or 3 ( 6 trials); hence, there were three distances to compare. Given that each item of distance data was based on a different number of trials, and distance 3 was only based on six items, median RTs were used in these analyses. Median latencies and accuracy percentages were calculated for each participant and condition after removing the trials that had been answered erroneously. Table 4 shows the median RTs and percentage of correct responses for each condition and math anxiety group.

Insert Table 4 about here 
A repeated measures ANOVA was performed with condition (congruent, neutral, incongruent distance 1 [d1], incongruent distance 2 [d2] and incongruent distance $3[\mathrm{~d} 3]$ ) as withinparticipant variables and math anxiety as a between-participants factor.

Low and high math-anxious individuals enumerated symbols at a similar speed, $F(1,53)<1$. There was a significant effect of condition, $F(4,212)=40.64, p<.001, \mathrm{y}_{\mathrm{p}}^{2}=.43$, which did not interact with math anxiety, $F(4,212)=1.15, p=.33, \mathrm{y}_{\mathrm{p}}^{2}=.021$. Bayesian analyses did not support the inclusion of anxiety as a predictor, with BF inclusion values of .41 and .13 for math anxiety and the interaction between math anxiety and condition.

Planned comparisons showed facilitation of congruent trials compared to neutral ones, $t(54)=9.51, p<.001$. Incongruent trials with a distance of 1 were answered more slowly than neutral ones, $t(54)=6.39, p<.001$. Incongruent trials with a distance of 2 between the result of enumeration and the digits displayed were also answered more slowly than neutral ones, $t(54)=5.51, p<.001$. More importantly, a distance effect was obtained, with $\mathrm{d} 1$ and $\mathrm{d} 2$ being significantly slower than $\mathrm{d} 3$ incongruent trials, $t(54)=5.02, p<.001$ and $t(54)=5.26, p<.001$ respectively. In contrast, there were no significant differences between RTs to trials of d1 and $\mathrm{d} 2, t(54)=1.07, p=.28$, nor between trials d 3 and neutral ones, $t(54)=1.16, p=.25$. Math anxiety did not interact with condition in any of the comparisons (all $\mathrm{ps} \geq .09$ and $\mathrm{BF}$ inclusion values ranging from .09 to .89 ).

The same analyses were conducted for the percentage of correct responses. Once again, the two groups performed similarly, $F(1,53)<1$. There was a main effect of condition, $F(4,212)=11.46$, $p<.001, \mathrm{y}_{\mathrm{p}}^{2}=.17$, that did not interact with math anxiety, $F(4,212)=1.13, p=.33, \mathrm{y}_{\mathrm{p}}^{2}=.021$. Planned comparisons paralleled the results obtained with latencies. Congruent trials were answered more accurately than neutral ones, $t(54)=3.58, p=.001$. Interference was also obtained, with distance 1 and distance 2 incongruent trials causing more errors than neutral ones, respectively $t(54)=5.32, p<.001$ and $t(54)=4.17, p<.001$. Once again, a distance effect was 
observed with $\mathrm{d} 3$ leading to less errors than $\mathrm{d} 1, t(54)=2.10, p=.04$.-However, $\mathrm{d} 1$ and $\mathrm{d} 2 \mathrm{did}$ not differ, $t(54)<1$, and neither did d 3 and d2trials, $t(54)=1.38, p=.17$. Math anxiety did not interact with condition in any of the comparisons (all ps>.13 and BF inclusion values ranging from .25 to .67).

To sum up, a distance effect was also obtained in this paradigm: response latencies and error rates were affected by the distance between numerosity and the identity of the Arabic digits to count. Thus, for instance, it took participants longer and they made more errors when they had to state that there were four threes rather than four ones. According to Pavese and Umiltà (1998), this effect would be located at the internal magnitude representation that had been activated by the two dimensions of the stimuli. Hence, although the identity of the Arabic digits was irrelevant for the task, it would have autonomously activated its numerical magnitude information in the compressed number line, causing more interference the closer it was to the numerosity to produce.

More importantly, the distance effect did not interact with math anxiety. HMA and LMA participants showed an equivalent increase in interference with symbolically close stimuli compared to farther ones. Given that the current experiment did not require comparison processes, our data support the claim by Dietrich et al. (2015) that their larger distance effects for HMA in symbolic comparison are not due to less precise magnitude representation, but to the comparison process per se.

\section{General Discussion}

Mathematics anxiety seems to be a condition with a higher prevalence than initially thought (Chang \& Beilock, 2016). This has led to a considerable increase in research on the factors that might influence the appearance or maintenance of maths anxiety. One result that has been reported repeatedly is that high math-anxious participants show longer distance effects in symbolic comparison (e.g. Maloney, Ansari \& Fugelsang, 2011). Given that distance effects have traditionally been considered a marker of access to internal magnitude representation, 
these authors concluded that math-anxious people might have less precise semantic representation of quantity. However, the latest research on the internal representation of magnitude has cast doubts on its unicity, and has suggested instead that symbolic and nonsymbolic formats might access (at least partially) different representations. In addition, the locus of distance effects has been debated, and it is now accepted that they can be located at the quantity representation, but also at response level.

The hypothesis of a link between math anxiety and less precise magnitude representation is attractive. It would naturally explain the worse performance of math anxious on a series of arithmetic tasks, it would establish nice parallelism with other conditions affecting number processing such as dyscalculia, and it would open the door to different forms of remediation (new or already existent), consisting of strengthening the mental number line. However, as stated above, the relationship between the distance effect and internal magnitude representation is currently under debate. The relationship between less acute number representation and a larger distance effect in math anxious must be confirmed before this hypothesis can be endorsed. An initial step in this direction was taken by Dietrich and collaborators (2015) who found, for the first time, no differences between HMA and LMA in a non-symbolic comparison task. Hence, they concluded that math anxiety was not related to a less precise abstract, modality-independent magnitude representation. In contrast, they replicated the larger distance effect previously found in HMA when the symbolic comparison was performed. Thus, math anxiety affected the processing of symbolic quantities only, but at what level? Dietrich et al. (2015) claimed that math anxiety affects comparison processes. HMA people tend to avoid arithmetic stimuli because they feel anxious about them. Therefore, they have less experience with numbers, and the connections between the symbolic representation of numbers and the "which numeral is larger" response are weaker. However, Dietrich et al. did not consider an alternative explanation; namely, that the impairment was located in the coding or representation of symbolic magnitudes. To discern between the magnitude representation and the response 
level alternatives we can use a task that cannot be solved by a simple comparison network, but in which the magnitude representation is accessed.

In this study, we asked participants to conduct a dot sets comparison task, an Arabic digits comparison task, and a counting Stroop. The first task replicated the results obtained by Dietrich and collaborators (2015): despite finding the typical ratio and congruency effects, HMA and LMA did not differ in terms of latencies, accuracy or $w$ fraction. Therefore, a relationship between math anxiety and acuity of non-symbolic magnitude representations does not seem to exist. We then examined the symbolic comparison where, unexpectedly, we failed to replicate the previous findings and the interaction between math anxiety and distance effect was far from significant. Lastly, the distance effect emerged again in the counting Stroop task, with close incongruent stimuli leading to longer latencies and more errors than far incongruent ones. Once again, though, the distance effect was equivalent for HMA and LMA participants. To sum up, after using two symbolic tasks in which magnitudes must be accessed we found no evidence that HMA participants had a less precise magnitude representation than their LMA peers. The findings of these three experiments are hard to reconcile with the idea that math anxiety is associated with acuity of the magnitude representation, either if we consider it as abstract, modality-independent or if specific coding of symbolic magnitudes is postulated.

As proposed by Dietrich et al. (2015), the possibility remains that math anxiety is related to impaired symbolic number comparison processes, and more specifically to the strength with which numbers evoke the relevant response in the comparison task. Nevertheless, this interpretation of their data must be treated with caution. First, the alleged effect of math anxiety was only found when measuring distance effects; there were no traces of it when comparing sizes. As reported in the introduction, Verguts and Opstal (2005), using a same-different task, described the opposite dissociation: they suggested that while the origin of the distance effect may be at the magnitude representation, size effects would be an indicator of the mapping between magnitude and response. Second, three different studies using a similar one-digit comparison task have found contradictory results: while Dietrich et al. (2015) found larger 
distance effects in HMA but equivalent effects of size, Núñez-Peña and Suárez-Pellicioni

(2014) found that both distance and size effects were only marginally larger in the HMA group.

In the current study, we found no differences whatsoever between HMA and LMA participants.

Third, Dietrich et al. (2015) already suggested that the larger distance effects found in symbolic comparison were due to variables other than math anxiety, albeit related to it. Those authors claimed that the less trained connections between the symbolic representation of the number and the response might be due to the lower motivation in math anxious individuals to work with numbers, or their tendency to avoid working with them. It has been reported that anticipation of a math task is perceived as a painful event in the brain of HMA individuals (Lyons \& Beilock, 2012). This finding fits neatly with the idea that this population has a low motivation to deal with numerical stimuli and tries to minimize contact with them. However, lack of motivation is not only linked to anxiety and may be caused, for instance, by previous instructional practices or the failure to perceive the utility of mathematics (Middleton \& Spanias, 1999). Since neither we nor the previous studies assessed motivation or attitudes toward mathematics in the two anxiety groups (high/low), these uncontrolled factors may explain the variability of patterns obtained in similar studies. We acknowledge this as a shortcoming of our work and suggest that future investigations should control the influence of these and other possible covariates such as state anxiety. Moreover, because of the simplicity of the tasks, we did not expect differences related to participants' mathematical skills. However, we cannot rule out the possibility that a slight advantage in the HMA group might have compensated the effects of math anxiety and thus favored the lack of group differences.

Lastly, another limitation of our study with regard to its generalization is the composition of the sample, which included only psychology students, $95 \%$ of whom were women.

Although it was not one of the aims of this study, we cannot conclude before saying a few words about another finding or, rather the lack of it. In recent years, it has been proposed that HMA and LMA might differ in their attentional control, with HMA showing less ability to resist the interference of irrelevant stimuli (e.g. Hopko, McNeil, Gleason \& Rabalais, 2002; 
Suárez-Pellicioni, Núñez-Peña \& Colomé, 2013, 2014). However, participants in the two mathanxious groups were similarly disturbed by the condition in which perceptual and numerical information were not correlated in the non-symbolic comparison task, and they showed equivalent congruency effects in the counting Stroop part. Hence, math-anxious participants did not show particular difficulties in ignoring irrelevant information across two different tasks. Although the current results might seem to contrast with previous literature, it is worth remembering that, according to Attentional Control Theory (Eysenck, Derakshan, Santos \& Calvo, 2007), anxiety affects processing efficiency to a greater extent than performance effectiveness. Thus, for instance, HMA participants in Suárez-Pellicioni, Núñez-Peña and Colomé (2013) showed a larger amplitude and latency of the P600/3b component, which indicates that processing implausible solutions had imposed heavier cognitive demands on them. However, their error rates or latencies did not differ from the LMA group. In this connection, a recent study by Pletzer, Kronbichler, Nuerk and Kerschbaum (2015) found that although HMA and LMA participants activated their default mode network differently during the comparison, they were matched for performance even in decade-unit incompatible trials in which incongruent information is presented.

To sum up, larger distance effects in high math-anxious individuals (HMA) performing comparison tasks were previously interpreted as indicating less precise magnitude representation in this population. Our study tested this hypothesis by asking two groups of HMA and LMA participants to conduct a non-symbolic comparison and two symbolic tasks. We found no evidence of less acute magnitude representation in HMA individuals. Hence, the relationship between math anxiety and the precision of the mental representation of either nonsymbolic or symbolic magnitudes is not supported. 


\section{REFERENCES}

Ansari, D. (2008). Effects of development and enculturation on number representation in the brain. Nature Reviews Neuroscience, 9(4), 278-291.

Alexander, L. \&Martray, C. (1989). The development of an abbreviated version of the Mathematics Anxiety Rating Scale. Measurement and Evaluation in Counseling and Development, 22, 143-150.

Ashcraft, M.H. \& Kirk, (2001). The relationship among working memory, math anxiety and performance. Journal of Experimental Psychology: General, 130(2), 224-237.

Clayton, S., Gilmore, C.K. \& Inglis, M. (2015). Dot comparison stimuli are not all alike: the effect of different visual controls on ANS measurement. Acta Psychologica, 161, 177-184. Cohen Kadosh, R., Cohen Kadosh, K., Kass, A., Henik, A. \& Goebel, R. (2007). Notationdependent and independent representations of numbers in the parietal lobes. Neuron, 53, 307314.

Chang, H. \& Beilock, S.L. (2016). The math anxiety-math performance link and its relation to individual and environmental factors: a review of current behavioral and psychophysiological research. Current Opinion in Behavioral Sciences, 10:33-38.

De Smedt, B., Verschaffel, L. \& Ghesquière, P. (2009). The predictive value of numerical magnitude comparison for individual differences in mathematics achievement. Journal of Experimental Child Psychology, 103 (4), 469-479.

DeWind, N.K. \& Brannon, E.M. (2016). Significant inter-test reliability across approximate number system assessments. Frontiers in Psychology, 7:310.

Dietrich, J.F., Huber, S., Moeller, K. \& Klein, E. (2015). The influence of math anxiety on symbolic and non-symbolic magnitude processing. Frontiers in Psychology, 6: 1621.

Dowker, A., Sarkar, A. \& Looi, C. Y. (2016). Mathematical anxiety: what have we learned in 60 years? Frontiers in Psychology, 7:508. 
Eysenck,M.W., Derakshan,N., Santos, R. \& Calvo, M.G.(2007). Anxiety and cognitive performance: Attentional control theory. Emotion, 7, 336-353.

Halberda, J., Mazzocco, M.M., \&Feigenson, L. (2008). Individual differences in non-verbal number acuity correlate with maths achievement. Nature, 455, 665-668.

Hembree, R. (1990). The nature, effects and relief of mathematics anxiety. Journal for Research in Mathematics Education, 21(1), 33-46.

Hock, H.S., \& Petrasek, J. (1973).Verbal interference with perceptual classification: the effect of semantic structure. Perception \& Psychophysics, 13, 116-120.

Hopko, D.R., Mahadevan, R., Bare, R.I., \& Hunt, M.K. (2003). The abbreviated math anxiety scale (AMAS) construction, validity, and reliability. Assessment, 10, 178-182.

Hopko, D.R., McNeil, D.W., Gleason,P.J., \& Rabelais, A.E. (2002). The emotional Stroop paradigm: performance as a function of stimulus properties and self-reported mathematics anxiety. Cognitive Therapy and Research, 26(2), 157-166.

Lyons, I. M., \& Beilock, S. L. (2012). Mathematics anxiety: Separating the math from the anxiety. Cerebral Cortex, 22, 2102-2110.

Maloney, E.A., Ansari, D. \& Fugelsang, J.A. (2011). The effect of mathematics anxiety on the processing of numerical magnitude. The Quarterly Journal of Experimental Psychology, 64(1), $10-16$.

Middleton, J.A. \& Spanias, P.A. (1999). Motivation for Achievement in Mathematics: Findings, Generalizations, and Criticisms of the Research. Journal for Research in Mathematics Education, 30 (1), 65-88.

Núñez-Peña, M. I.,\& Suárez-Pellicioni, M. (2014). Less precise representation of numerical magnitude in high math-anxious individuals: an ERP study of the size and distance effects. Biological Psychology, 103, 176-183. 
Núñez-Peña, M. I., Suárez-Pellicioni, M., Guilera, G., \&Mercadé-Carranza, C. (2013). A Spanish version of the short Mathematics Anxiety Rating Scale (sMARS). Learning and Individual Differences, 24, 204-210.

Pavese, A. \& Umiltà, C. (1998). Symbolic distance between numerosity and identity modulates stroop-like interference. Journal of Experimental Psychology: Human Perception and Performance, 24(5), 1535-1545.

Piazza, M., Pinel, Ph., Le Bihan, D. \& Dehaene, S. (2007). A magnitude code common to numerosities and number symbols in human intraparietal cortex. Neuron, 53, 293-305.

Pletzer, B., Kronbichler,M., Nuerk, H-C, Kerschbaum, H.H. (2015). Mathematics anxiety reduces default mode network deactivation in response to numerical task. Frontiers in Human Neuroscience, 9:202.

Price, G., Palmer,D., Battista, Ch.\& Ansari, D. (2012). Nonsymbolic numerical magnitude comparison: Reliability and validity of different task variants and outcome measures and their relationship to arithmetic achievement in adults. Acta Psychologica, 140, 50-57.

Richardson, F.C. \& Suinn, R.M. (1972). The mathematics anxiety rating scale: Psychometric data. Journal of Counseling Psychology, 19(6), 551-554.

Rousselle, L. \& Noèl, M.P. (2007), Basic numerical skills in children with mathematical learning disabilities: A comparison of symbolic vs. non-symbolic number magnitude processing. Cognition, 102, 361-395.

Spielberger, C. D., Gorsuch, R., \&Lushene, R. (2008). Cuestionario de ansiedad Estado-Rasgo, STAI. Madrid: TEA Ediciones.

Spielberger, C. D., Gorsuch, R., Lushene, R., Vagg, P. R., \& Jacobs, G. A. (1983). Manual for the State-Trait anxiety inventory. Palo Alto, CA: Consulting Psychologists Press. 
Suárez-Pellicioni, M., Núñez-Peña, M. I, \& Colomé, À. (2013). Mathematical anxiety effects on simple arithmetic processing efficiency: an event-related potential study. Biological Psychology, 94, 517-526.

Suárez-Pellicioni, M., Núñez-Peña, M. I, \& Colomé, À. (2014). Reactive recruitment of attentional control in math anxiety: an ERP study of numeric conflict monitoring and adaptation. PLOS ONE, 9(6), e99579.

Suárez-Pellicioni, M., Núñez-Peña, M. I, \& Colomé, À. (2016). Math anxiety: A review of its cognitive consequences, psychophysiological correlates, and brain bases. Cognitive, Affective \&Behavioural Neuroscience, 16(1),3-22.

Van Opstal, F., Gevers, W., De Moor, W. \&Verguts, T. (2008). Dissecting the symbolic distance effect: comparison and priming effects in numerical and nonnumerical orders. Psychonomic Bulletin \& Review, 15(2), 419-425.

Van Opstal, F. \&Verguts, T. (2011). The origins of the numerical distance effect: the samedifferent task. Journal of Cognitive Psychology, 23(1), 112-120.

Verguts, T. \& Fias, W. (2004). Representation of number in animals and humans: a neural model. Journal of Cognitive Neuroscience, 16, 1493-1504.

Verguts, T., Fias, W. \& Stevens, M. (2005). A model of exact small-number representation. Psychonomic Bulletin \& Review, 12 (1), 66-80.

Verguts, T. \& Van Opstal, F. (2005). Dissociation of the distance effect and size effect in onedigit numbers. Psychonomic Bulletin \& Review, 12 (5), 925-930. 
Table 1. $w$ values and mean RTs, standard deviations (in brackets) and percentage of hits $(\% \mathrm{H})$ for each congruency condition and ratio for the LMA and HMA groups.

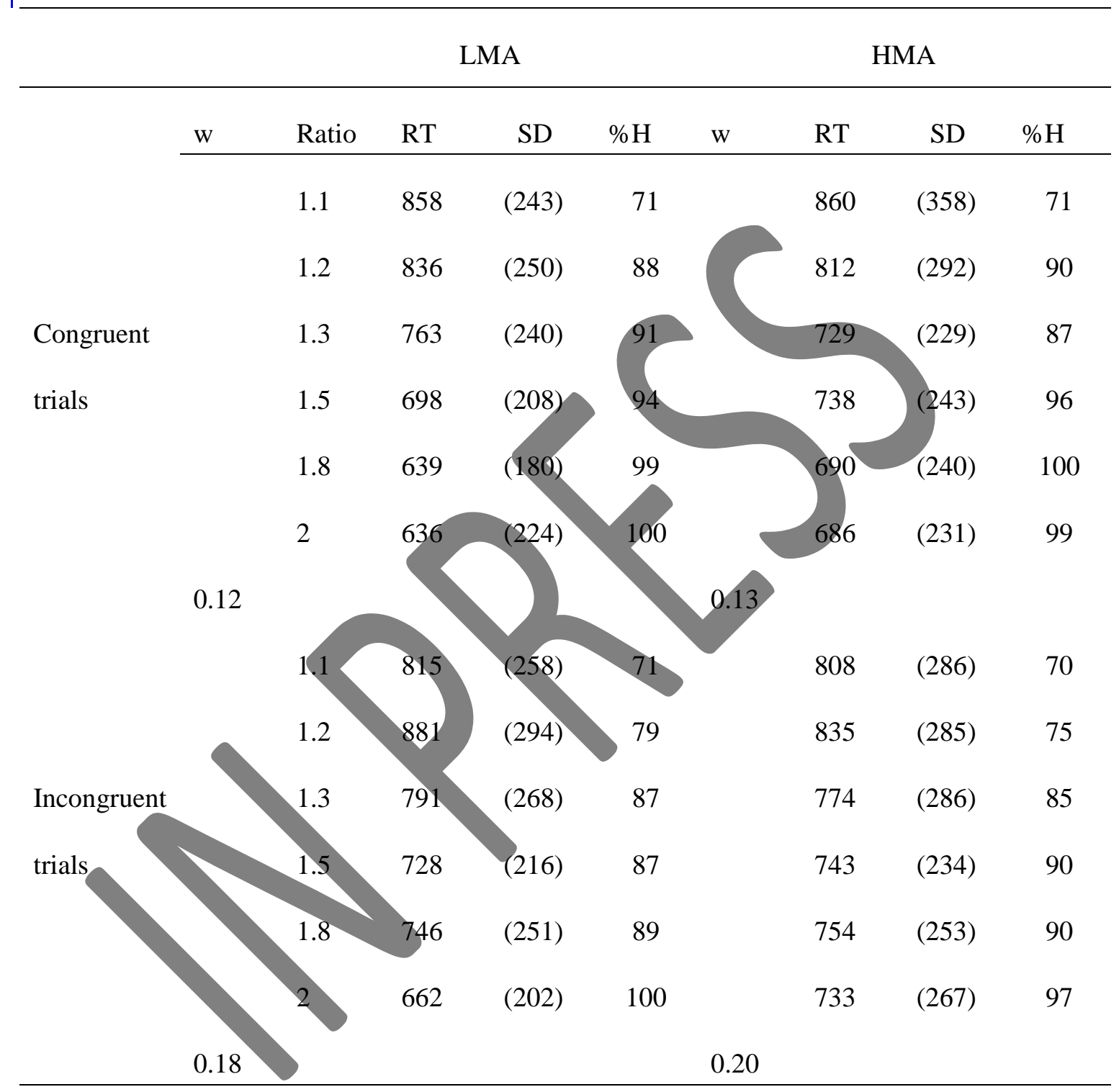

Note: Congruent trials: The total area of each color is proportional to the number of dots.

Incongruent: anti-correlated trials in which the total area filled by the set with less dots is larger than the area of the set with more dots. 
Table 2. Stimuli presented in Experiment 1B according to the size of the pair and the distance between the digits.

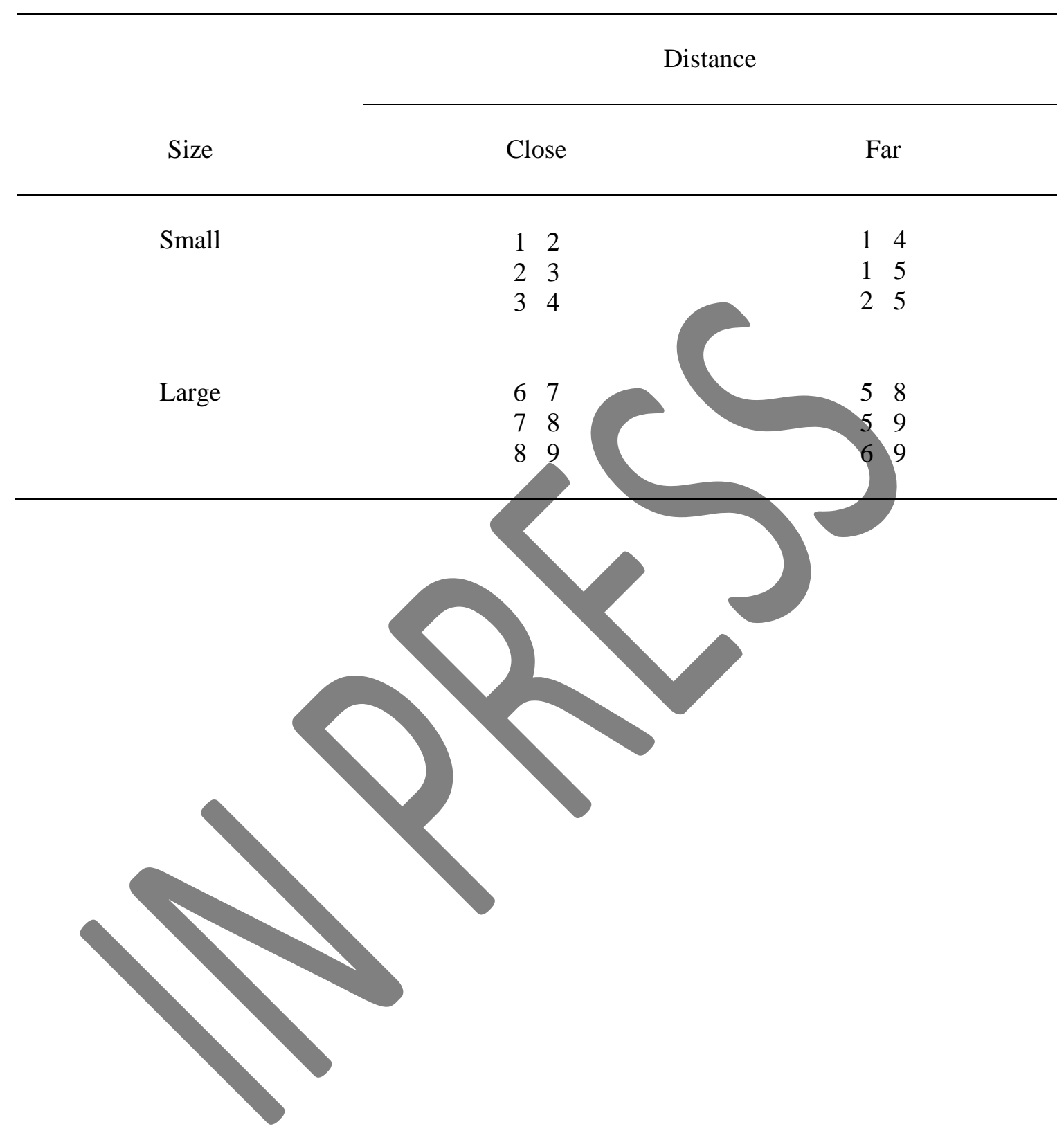


Table 3. Mean RTs, standard deviations (in brackets) and percentage of hits $(\% \mathrm{H})$ for each distance and size for the LMA and HMA groups.

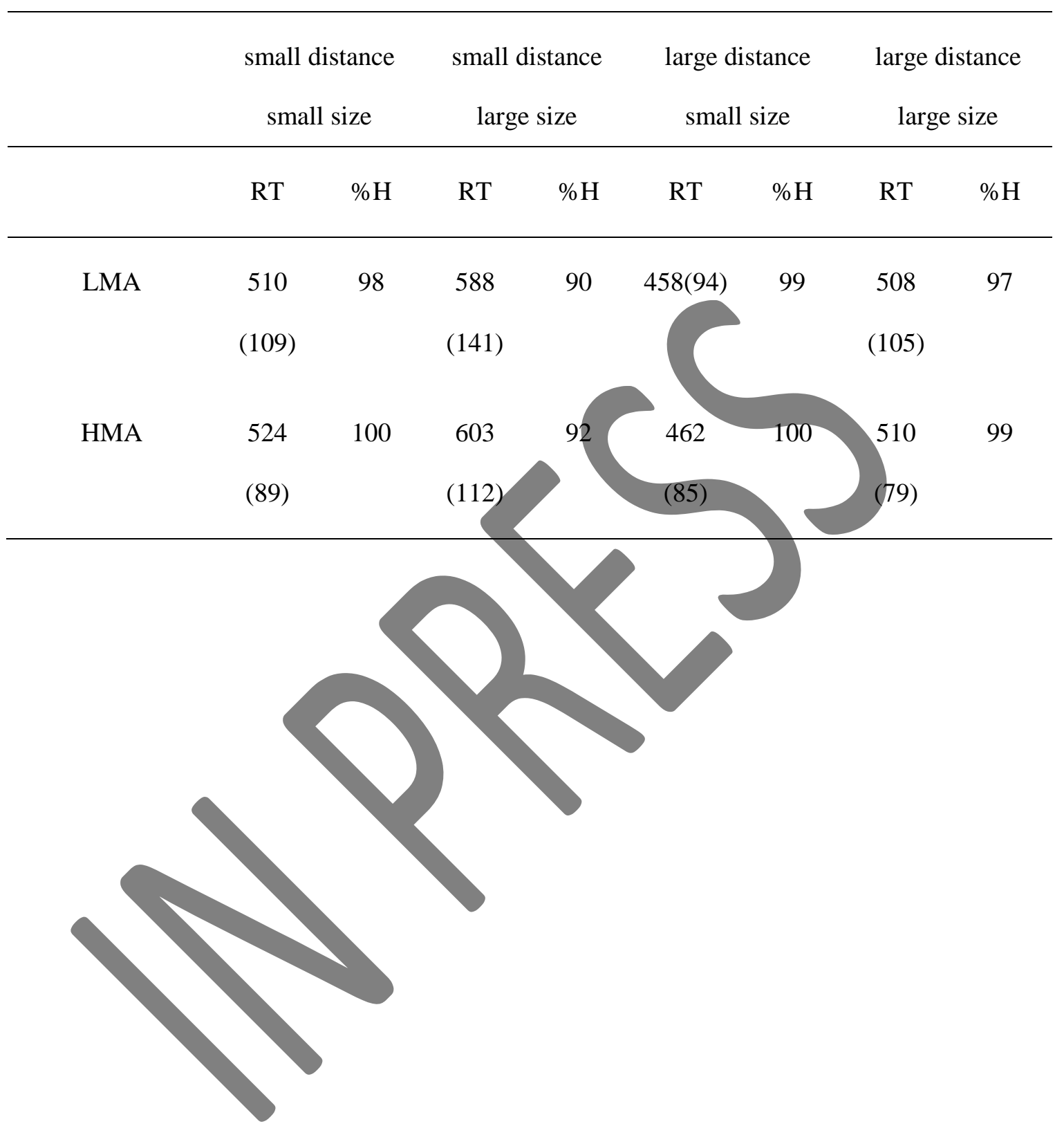


Table 4. RTs (mean of medians), standard deviations (in brackets) and percentage of hits $(\% \mathrm{H})$ for each condition for the LMA and HMA groups.

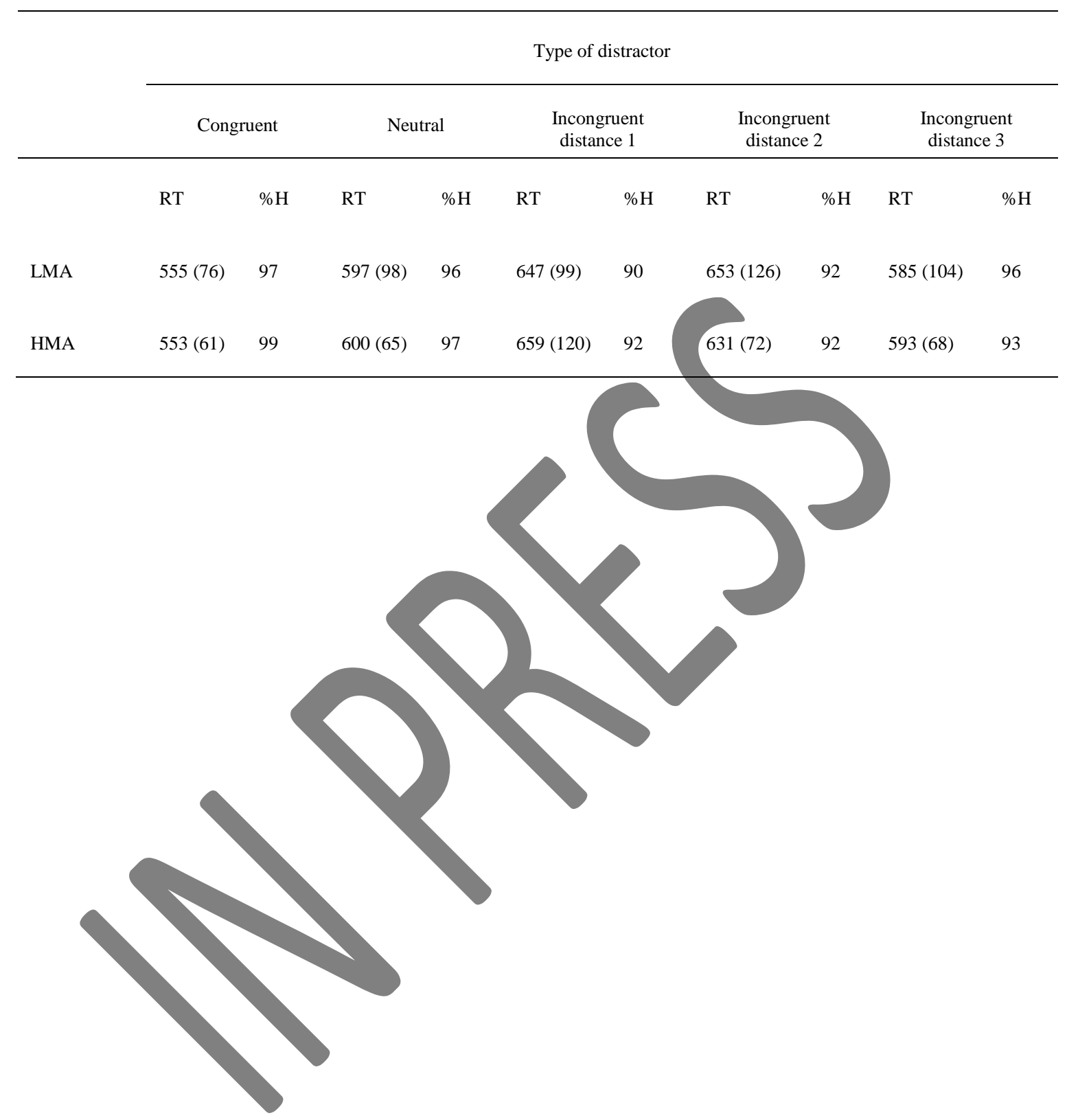

